\title{
Optimization of Fluconazole Pickering Emulsion Using Taguchi Orthogonal Array Design
}

\author{
D. Prasanthi, N. Varsha Priya, Amoolya Chennuri and P.K. Lakshmi \\ G. Pulla Reddy College of Pharmacy, Mehdipatnam, Hyderabad-500028, Telangana State, India \\ (Received: February 06, 2019; Accepted: November 28, 2020; Published (web): December 10, 2020)

\begin{abstract}
Emulsifier-free, particle-stabilised emulsions called Pickering emulsions of fluconazole for topical delivery, were studied using Taguchi L9 orthogonal array (OA) design. Formulations were prepared by homogenization method, using bentonite in the concentration range of $2-4 \% \mathrm{w} / \mathrm{v}$, oil phase (coconut oil, oleic acid, sesame oil). In Taguchi L9 OA experimental design, independent variables (oil phase \& concentration of bentonite) effect on dependent variables (drug content \& release rate) was studied. All the formulations have shown good physicochemical properties. The PS3 formulation containing sesame oil and $4 \%$ bentonite was optimized as particle size was found to be $758.0 \mathrm{~nm}$, zeta potential $-48 \mathrm{mv}$, with $Q_{8}$ of $166.70 \pm 0.15 \mu \mathrm{g} / \mathrm{cm}^{2}$, flux of $17.33 \pm 0.02 \mu \mathrm{g} / \mathrm{cm}^{2} / \mathrm{hr}$, permeability coefficient of $3.46 \pm 0.015 \mathrm{~cm} / \mathrm{hr} \times 10^{-3}$ and skin content of $4.01 \pm 0.24 \mathrm{mg} / \mathrm{g}$. The formulations were stable and non-irritant. Hence pickering emulsion of fluconazole using sesame oil can be used for topical delivery as the drug release was maintained at the localised site.
\end{abstract}

Key words: Pickering emulsion, fine solid particles, skin permeation, Taguchi orthogonal array

\section{INTRODUCTION}

In 1907, Pickering discovered adsorption of fine particles at interfaces of liquid-liquid or liquid-gas, resulting in monomolecular layer on dispersed globules, thus stabilizing emulsions. Fine particles or surfactants added, result in long-term stability, as they accumulate at fluid-fluid interface in emulsions forming a layer over dispersed globules/droplets, inhibiting drop recombination and coarsening. Particles bound on surface are irreversible. Emulsifier-free emulsions are called pickering emulsions, in which fine particles have substituted the classical nanometric/micrometric size particles which adsorb at interface of oil-water. ${ }^{1}$

Silica, clay and latex are some examples of fine solid particles used in pickering emulsion as emulsifiers. Pickering emulsions have advantages of remarkable stability against coalescence and are also stable to changes in $\mathrm{pH}$, salt concentration and temperature during processing.

Correspondence to: D. Prasanthi

E-mail: prasanthidhanu@gmail.com

Dhaka Univ. J. Pharm. Sci. 19(2): 169-178, 2020 (December)

DOI: https://doi.org/10.3329/dujps.v19i2.50633
Various parameters of fine solid particles, like particle concentration, particle wettability, shape and size are found to be effective in stabilizing emulsions, by many researches. Adsorption of fine solid particles as dense shell around droplets acts as barrier against material transfer. ${ }^{2,3}$

The objective of present study is to optimize pickering emulsions of fluconazole for enhanced topical delivery using Taguchi L9 orthogonal array design. Fluconazole is a first-generation triazole antifungal medication, used for fungal or yeast infections like vaginal candidiasis and other candida infections (including urinary tract infections, or fungal infections like cryptococcal meningitis). Its mechanism of action includes interaction with $14-\alpha$ demethylase, a cytochrome P-450 enzyme which is necessary in conversion of lanosterol to ergosterol. ${ }^{4}$

In the present study, Pickering emulsions were prepared by homogenization method and compared over surfactant-based emulsion (SBE) for permeation enhancement. The optimized formulation was evaluated for entrapment efficiency, droplet size, zeta 
potential, surface morphology, in-vitro diffusion study, ex-vivo permeation study, skin content, skin irritation study and stability study.

\section{MATERIALS AND METHODS}

Fluconazole was gifted by Aurobindo Pharma Ltd. Coconut oil and sesame oil were purchased from local market. Oleic acid, Span 60 and Tween 20 were procured from S.D. Fine Chem. Ltd. (India).

Experimental design. Taguchi L9 orthogonal array experimental design with two-factors, threelevels was constructed using MINITAB 17 software (Minitab Inc., PA, USA). Type of oil phase and concentration of particulate emulsifier (Bentonite) were selected as independent variables and responses (entrapment efficiency, percentage drug release) as dependent variables which are shown in Table 1.
Preparation of Pickering emulsion. Pickering emulsions were prepared by Homogenisation method using high speed disperser. Finely divided solid particles of bentonite at various concentrations of 2$4 \%$ as specified in Table 2 were dispersed in water $(10 \mathrm{ml})$ for $15 \mathrm{mins}$ using homogenizer at $1000 \mathrm{rpm}$. Fluconazole $(0.5 \%)$ was dispersed into the oil phase (oleic acid, coconut oil, sesame oil) and then aqueous phase (oil: aqueous phase ratio of 1:9) was added to it at $1300 \mathrm{rpm}$ for $60 \mathrm{mins}$. Then aqueous solid particles were dispersed to the above dispersion with continuous stirring at $1300 \mathrm{rpm}$ for 60 mins. ${ }^{5}$ Finally volume was made up to $25 \mathrm{ml}$ with deionized water. Resultant emulsion was stirred well for additional 10 minutes by maintaining the system at room temperature. Nine formulations were prepared according to Taguchi L9 OA design. Pickering emulsions were compared with surfactant-based emulsion (SBE).

Table 1. Two-factor, three-level Taguchi L9 Orthogonal array experimental design.

\begin{tabular}{|c|c|c|c|}
\hline \multirow[t]{2}{*}{ Factors } & \multicolumn{3}{|c|}{ Levels } \\
\hline & 1 & 2 & 3 \\
\hline A. Type of oil phase & Oleic acid & Coconut oil & Sesame oil \\
\hline $\begin{array}{l}\text { B. Concentration of } \\
\text { particulate emulsifier }(\%)\end{array}$ & 2 & 3 & 4 \\
\hline
\end{tabular}

Response: Entrapment efficiency and percentage drug release.

Table 2. Taguchi L9 orthogonal array experimental design for Pickering emulsion.

\begin{tabular}{llccc}
\hline $\begin{array}{l}\text { Formulation } \\
\text { code }\end{array}$ & \multicolumn{2}{c}{ Independent variables } & \multicolumn{2}{c}{ Dependent variables } \\
\cline { 2 - 5 } & $\mathrm{A}$ & $\mathrm{B}(\%)$ & $\mathrm{Y} 1(\%)$ & $\mathrm{Y} 2(\%)$ \\
\hline PO1 & 1 & 1 & $81.07 \pm 0.152$ & $18.1 \pm 0.02$ \\
$\mathrm{PO} 2$ & 1 & 2 & $84.16 \pm 0.25$ & $14.9 \pm 0.04$ \\
$\mathrm{PO} 3$ & 1 & 3 & $91.2 \pm 0.12$ & $17.0 \pm 0.03$ \\
PC1 & 2 & 1 & $79.89 \pm 0.18$ & $17.8 \pm 0.04$ \\
PC2 & 2 & 2 & $86.48 \pm 0.24$ & $18.7 \pm 0.03$ \\
PC3 & 2 & 3 & $88.48 \pm 0.32$ & $19.1 \pm 0.03$ \\
PS1 & 3 & 1 & $81 \pm 0.25$ & $19.3 \pm 0.05$ \\
PS2 & 3 & 2 & $82.08 \pm 0.35$ & $21.4 \pm 0.05$ \\
PS3 & 3 & 3 & $93.7 \pm 0.42$ & $32 \pm 0.03$ \\
\hline A: Typp
\end{tabular}

A: Type of oil phase; B: Concentration of bentonite (\%), Y1: Entrapment efficiency (\%); Y2: Percentage drug release. Values represent mean $\pm S D(n=3)$.

SBE classical emulsion was prepared using $1.5 \mathrm{~g}$ of sesame oil as oil phase and Tween $20(0.19 \mathrm{gm})$ and Span $60(0.8 \mathrm{gm})$ as surfactants. The surfactants were heated at $80^{\circ} \mathrm{C}$ separately. Then to Span 60 , oil phase was added and to Tween 20, aqueous phase was added. The two phases were allowed to cool. 
Fluconazole $(0.5 \%)$ was added in oil phase and dispersed. Then oil phase was added into aqueous phase and gently shaken.

The resultant Pickering emulsion formulations were studied for drug content, physicochemical properties, in vitro diffusion study and ex vivo permeation study.

Solubility studies. $2 \mathrm{ml}$ of oil was taken and the drug was added to it until a supersaturated solution is attained. This solution was transferred into Eppendorf tubes and placed in orbital shaker for $24 \mathrm{hrs}$. The supernatant was filtered using Whatman filter paper, diluted with phosphate buffer saline at $\mathrm{pH} 7.4$ and analyzed for drug content at $263 \mathrm{~nm}$.

\section{Determination of type of emulsion}

Dilution test. The type of emulsion was determined by diluting the continuous phase with oil and water. If a stable emulsion results on dilution with water then it is oil in water emulsion. If it is unstable with water dilution but stable with oil dilution then it is water in oil emulsion.

Dye-solubility test. In this test, the type of emulsion is identified by mixing the emulsion with a water-soluble dye. Then the emulsion is observed under microscope. If the continuous phase appears colored, then it is oil in water type but if the scattered globules appear colored and the continuous phase colorless, then it is water in oil type.

Fluorescence test. The type of emulsion can be identified by exposing the emulsion to ultra-violet radiations and observing it under microscope for fluorescence. If continuous fluorescence is observed, then it is water in oil type emulsion and if only spotty fluorescence is seen then it is oil in water type emulsion.

Conductivity test. This test is based on conduction of electricity by water which is a good conductor. This test involves an assembly with a pair of electrodes connected to a lamp. To identify the type of emulsion, the electrodes are dipped in the emulsion and if the lamp glows then it is oil in water type emulsion, if lamp doesn't glow then it is water in oil type emulsion.
Entrapment efficiency. The amount of drug entrapped within oil phase is evaluated by taking one $\mathrm{ml}$ of formulation into an Eppendorf tube, and it was centrifuged at 5,000 rpm for $15 \mathrm{mins}$ in two cycles. The supernatant which represents unentrapped drug was collected, and determined by UV-VIS spectrophotometer at $263 \mathrm{~nm}^{6}$

The percentage of drug entrapment was calculated as $\left(C_{t}-C_{f}\right) / C_{t}$, where $C_{t}$ is the total (entrapped and unentrapped) concentration of fluconazole and $\mathrm{C}_{\mathrm{f}}$ is the concentration of unentrapped fluconazole.

Drug content. $1 \mathrm{ml}$ of emulsion was transferred into $50 \mathrm{ml}$ conical flask and dissolved in $10 \mathrm{ml}$ of phosphate saline buffer ( $\mathrm{pH}$ 7.4). The conical flask was placed in orbital shaker for $2 \mathrm{hrs}$. Then the solution was centrifuged for $15 \mathrm{mins}$ at $5,000 \mathrm{rpm}$. The solution was filtered, diluted and drug content was measured spectrophotometrically at $263 \mathrm{~nm}$.

Determination of viscosity. The viscosity was determined using Brookfield Viscometer LVDVII+PRO. The formulations were taken in a beaker and kept aside for $30 \mathrm{~min}$ at $30 \pm 1^{\circ} \mathrm{C}$. Then the viscosity was determined using spindle no 64, at $50 \mathrm{rpm}$. Care was taken that spindle doesn't touch the bottom as it damages the spindle.

Droplet size and zeta potential. The droplet size of all formulations was determined by microscopy method (triangular research microscope with Fuji film digital camera). The optimized formulation droplet size and zeta potential were measured by photon correlation spectroscopy (Delsa Nano, Beckman Coulter Inc. UK).

Surface morphology. Surface morphology of the prepared vesicles was characterized by Scanning electron microscopy (SEM). Sample was mounted on a clear-glass stub, air-dried, gold coated with Polaron E5100 sputter coater (Polaron, United Kingdom), and visualized under scanning electron microscope (S4100, Hitachi, Shiga, Japan).

In vitro diffusion studies. Locally fabricated Franz diffusion cell with receptor volume of $20 \mathrm{ml}$ was used. Dialysis membrane was used as barrier to mimic skin and it was mounted between donor and receptor compartments. Emulsion sample equivalent 
to $5 \mathrm{mg}$ of drug was taken in donor compartment and receptor compartment was filled with PBS (pH 7.4). The hydrodynamics in the compartment were maintained at $300 \mathrm{rpm}$ by stirring on a magnetic stirrer. At predetermined time intervals $1 \mathrm{ml}$ of sample was withdrawn and replaced with equal volume of buffer. The samples were analyzed for drug content spectrophotometrically at $263 \mathrm{~nm}$ with appropriate dilution.

Ex vivo permeation study. Study approved by Institutional animal ethical committee (IAEC) using rat as model was followed. Franz diffusion cells were locally fabricated with an area of $4.9 \mathrm{~cm}^{2}$ of opening in donor \& receptor compartment and $20 \mathrm{ml}$ receptor volume. The thawed rat skin was mounted onto diffusion cell in between donor and receptor compartment such that dermis side was in constant contact with receptor solution. Formulation was applied to stratum corneum facing the donor compartment and the hydrodynamics in the receptor compartment was maintained at $300 \mathrm{rpm}$ by stirring on magnetic stirrer. $1 \mathrm{ml}$ of sample was withdrawn for $8 \mathrm{hrs}$ at predetermined time intervals and drug content was analyzed at $263 \mathrm{~nm}$ by UV-VIS double beam spectrophotometer.

Calculation of permeability parameters. Parameters were calculated in this part of the study to compare the drug transfer and permeation properties amongst the tested formulae. These parameters include steady state flux (Jss), lag time, permeability coefficient $(\mathrm{Kp})$ and cumulative amount permeated $\left(\mathrm{Q}_{8}\right)^{7}{ }^{7}$

Steady state flux. The rate of diffusion or transport of a substance through a permeable membrane is defined as Flux. After reaching the steady state concentration of drug permeated, flux was calculated using the following equation:

$$
J \boldsymbol{s} \boldsymbol{s}=d M / S . d t
$$

where, Jss- Steady state flux; dM- amount of drug permeated; S- unit cross-section area of skin to which formulation is applied and t- time. Flux is calculated by plotting cumulative amount permeated per square centimeter of area on y axis and time on $\mathrm{x}$ axis. Slope of the steady state portion of curve gives flux.
Lag time: X- intercept of steady state portion of curve, which is obtained by extrapolating the steady state portion of curve onto $\mathrm{x}$ axis gives lag time.

Permeability coefficient $(\mathbf{c m} / \mathbf{h r})$. The permeability coefficient $(\mathrm{Kp})$ was calculated with the following equation:

$$
\mathrm{Kp}=\frac{J s s}{\boldsymbol{C V}}
$$

where, $\mathrm{CV}$ is the total donor concentration of the formulation.

Cumulative amount permeated $\left(\mathbf{Q}_{8}\right)$. It is cumulative amount of drug permeated through skin per exposed area of skin at regular time intervals. The subscript represents hour, $8^{\text {th }}$ hour reading is $\mathrm{Q}_{8}$. It is calculated from drug permeated at regular time intervals against area of skin exposed to formulation.

Skin content. Drug retained in the skin after 8 hours of study was determined as skin content. The exposed skin after study was removed from diffusion cell, washed with methanol, cut into small pieces, $4 \mathrm{ml}$ of methanol and $6 \mathrm{ml}$ of $\mathrm{pH} 7.4$ phosphate buffer saline was added and homogenized. After homogenization the mixture was centrifuged at $5000 \mathrm{rpm}$ for $30 \mathrm{~min}$, filtered through Whatman filter paper and analyzed for drug content with appropriate dilutions by UV-VIS double beam spectrophotometer at $263 \mathrm{~nm}$

Calculation of release kinetics for prepared Pickering emulsions. Model dependent kinetics of the dosage form was used to analyze the mechanism of the drug release. The amount of drug permeated at regular intervals was fitted into zero-order release equation, first order release equation, Higuchi equation and Korsmeyer-Peppas release model.

Zero-order: $\mathrm{Q}_{\mathrm{t}}=\mathrm{Q}_{0}+\mathrm{K}_{0} \mathrm{t}$;

First order: $\ln \mathrm{Q}_{\mathrm{t}}=\ln \mathrm{Q}_{0}+\mathrm{K}_{1} \mathrm{t}$;

Higuchi: $\mathrm{Q}_{\mathrm{t}}=\mathrm{K}_{\mathrm{H}} \mathrm{t}^{1 / 2}$;

Korsmeyer-Peppas: $\mathrm{Q}_{\mathrm{t}} / \mathrm{Q}_{\infty}=\mathrm{K}_{\mathrm{k}} \mathrm{t}_{\mathrm{n}}$;

Where $\mathrm{Q}$ represents cumulative amount permeated, $\mathrm{t}$ time and $\mathrm{K}_{0}, \mathrm{~K}_{1}, \mathrm{~K}_{\mathrm{H}}, \mathrm{K}_{\mathrm{k}}$ represent release rate constant of zero order, first order, Higuchi and Korsemeyer Peppas respectively. 
For all the formulations Regression coefficients (r) and release component " $n$ " were calculated to determine the type of release kinetics. The model dependent kinetic calculations were carried out in Excel-MS Office.

Fourier transforms infrared spectroscopic analysis of drug and excipients. Drug-excipient compatibility was studied. Fourier transform infrared spectra were obtained for pure drug fluconazole using the potassium bromide disc method, by preparing pellet with dry samples (applying pressure of 10 tons/inch ${ }^{2}$ for $10 \mathrm{~min}$ ). Liquid FT-IR was carried out for the prepared formulation and their compatibility was checked.

Skin irritation study. It was performed by using rabbit animal model. Three rabbits were used for study. The formulations control, standard skin irritant, placebo and test were applied on the left and right dorsal surface of rabbit skin and were examined for 8 hours. Erythema and edema were evaluated and the score was given according to the primary dermal irritation index classification (PDII). ${ }^{8}$

Stability studies. Physical stability of optimized formulation was evaluated. The optimized pickering emulsion was sealed in $10 \mathrm{ml}$ glass vials and stored for two months at room temperature. Every month entrapment efficiency was determined.

\section{RESULTS AND DISCUSSION}

Pickering emulsions were prepared by homogenisation method using high speed dispersers according to formulations given by Taguchi L9 OA experimental design given in Table 2. Initially the solubility of the drug was tested in different oil phases used in the formulation. A maximum solubility of $0.127 \mathrm{mg} / \mathrm{ml}$ was determined in oleic acid when compared to coconut oil $0.036 \mathrm{mg} / \mathrm{ml}$ and sesame oil $0.0596 \mathrm{mg} / \mathrm{ml}$. The formulations were tested for the type of emulsion by dilution test, dye test, fluorescence test and conductivity test. Identification tests indicated oil in water type of emulsion. The entrapment efficiency was in the range of $81.07 \pm 0.15 \%$ to $93.7 \pm 0.42 \%$. Formulation PS3 i.e., sesame oil showed maximum entrapment efficiency of $93.7 \pm 0.42 \%$. The drug content of the formulated Pickering emulsion was estimated spectrophoto-metrically at $263 \mathrm{~nm}$. The results were within the official limits ranging from $97.90 \pm 0.2 \%$ to $99.62 \pm 0.2 \%$. Viscosity of Pickering emulsions was determined using Brookfield viscometer LVDVII+PRO. Initially 5\% solution of Pickering emulsions were prepared and viscosity was determined at 50 rpm using spindle no 64 at temperature $30^{\circ} \mathrm{C}$ and torque $32 \%$. The viscosity of the formulations ranged between $485 \pm 15$ cps to $569 \pm 30$ cps. Viscosity of PO3 formulation was higher than other formulations as oleic acid is highly viscous than coconut and sesame oil. Droplet size of Pickering emulsions by microscopy method ranged from $105 \mu \mathrm{m}$ to $140 \mu \mathrm{m}$. The Zeta potential of optimized formulation PS3 was negative $(-48 \mathrm{mV})$ indicating the formulation is stable. The droplet size of optimized formulation PS3 was $758 \mathrm{~nm}$. Surface morphology and the threedimensional nature of the emulsion was studied through SEM images given in Figure 1. The images confirmed the presence of oil droplets as a rigid surface possessing elliptical shape.

In vitro diffusion studies were performed with Franz diffusion cells using dialysis membrane. The in vitro drug release result is given in Table 2 as percentage drug release. The formulation PS3 (sesame oil with $4 \%$ bentonite) has shown $32 \pm 0.03 \%$ of drug release in $8 \mathrm{hrs}$ respectively. As sesame oil has high penetration ability than that of coconut oil, so it is easily permeated through the epidermal layer of the skin. The drug release rate was in the order of sesame oil > coconut oil > oleic acid. The release rate was found to be $97.20 \mu \mathrm{g} / \mathrm{cm}^{2} / \mathrm{hr}^{-1}$ for the optimized formulation (PS3). The optimized Pickering emulsion is compared with that of the classical emulsion (SBE) possessing the surfactants. The drug release of classical emulsion (SBE) $(52.8 \pm 0.02 \%)$ is higher than the Pickering emulsion $(32 \pm 0.03 \%)$, due the presence of surfactants which increase the permeation of the drug. The decrease in permeation of Pickering emulsion is due to the presence of particulate emulsifier which gets adsorbed on to oil droplets and causes the deposition of the emulsion in the 
epidermal layer, thus causing the slow release of drug and at the target site. ${ }^{5}$

Taguchi OA L9 design of experiment was used for optimising pickering emulsions by varying type of oil phase and concentration of bentonite. Results are given in Table 2. The effect of independent variables on dependent variables was evaluated by signal to noise (SN) ratio plots. The desirable effects are considered as signal and undesirable effects as noise. Software gives the overall effect of the independent variables on responses which are dependent variables in the form of $\mathrm{SN}$ ratio plots. SN ratio plots for the responses are given in Figure 2 and Figure 3. Main effects plot of SN ratios showed type of oil phase had no significant effect on entrapment efficiency and percentage drug release ( $p>0.05)$. But maximum permeation was observed with sesame oil as it offers high penetration through the skin layer. Concentration of bentonite (2\% to $4 \%$ ) effected entrapment efficiency and flux. From the SN ratios plot it has been observed entrapment efficiency and percentage drug release increased with increase in bentonite concentration up to $3 \%$, but further increase decreased entrapment efficiency and percentage drug release. This effect is not significant ( $p>0.05$ ).

When the effect of one factor depends on the level of the other factor, one can use an interaction plot to visualize possible interactions. In this plot, Xaxis represents concentration of particulate emulsifier and Z-axis represents type of oil phase. Figure 4 shows an interaction between type of oil phase and the concentration of particulate emulsifier which was detected by the absence of parallel lines. The interaction had an effect on the entrapment efficiency. As the concentration of emulsifier increases, entrapment efficiency increased. From Figure 4, we can infer that there is an interaction between the oil phase and at low concentration of particulate emulsifier. There was no interaction between the oil phase and particulate emulsifier as the concentration of particulate emulsifier increased. We can determine that the type of oil phase has certain influence on the drug release when compared to the concentration of particulate emulsifier. From both plots we can determine that the two factors have a simultaneous influence in the release rate of the Pickering emulsions.
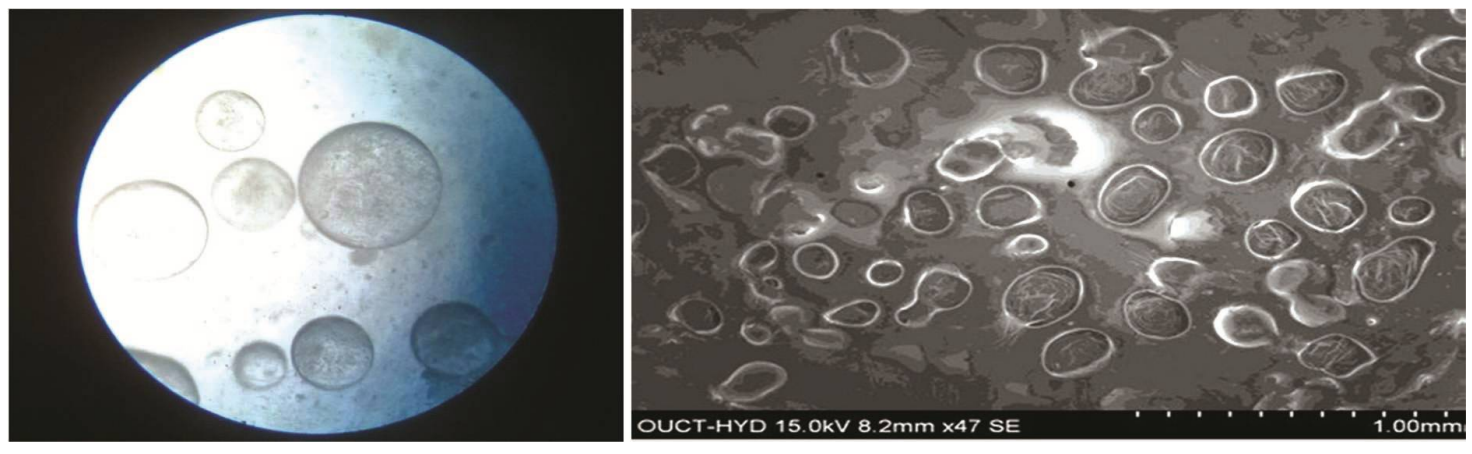

Figure 1. Photomicrograph and SEM analysis of Pickering emulsion.

Ex vivo permeation studies were performed using rat abdominal skin. The optimized formulation showed maximum drug release rate of 63.19 $\mu \mathrm{g} / \mathrm{cm}^{2} / \mathrm{hr}$ for $8 \mathrm{hrs}$ when compared to classical emulsion having drug release rate of $304.9 \mu \mathrm{g} / \mathrm{cm}^{2} / \mathrm{hr}$ for $8 \mathrm{hrs}$. Upon comparing with classical emulsion, the permeability of Pickering emulsion was low which is required. As Pickering emulsion is a topical preparation, the effect should be local and the drug should be deposited into the epidermal layer of the skin and provide a drug release at specific site. Previous study reported that Pickering emulsions (retinol used as a model drug) increased accumulation of drug in stratum corneum when compared to surfactant-based emulsions. Hence, they can be used as vehicles for topical application. ${ }^{9}$ As 
the Pickering emulsion formulation is able to retain the lipophilic ingredients in the outer layers of skin, formulation can be potentially used to target drugs to the skin surface. ${ }^{10}$

Upon comparing the ratio of permeability of drug, the PS3 formulation with sesame oil and $4 \%$ bentonite was optimized as it showed low permeation and high skin deposition which is required for the topical delivery of Pickering emulsions. This is due to the adsorption of bentonite particle across the oil droplets and may be due to the lack of flexibility of the droplets. The PO3 formulation has shown high release than PS3 (Figure 5), because oleic acid itself acts as a penetration enhancer.

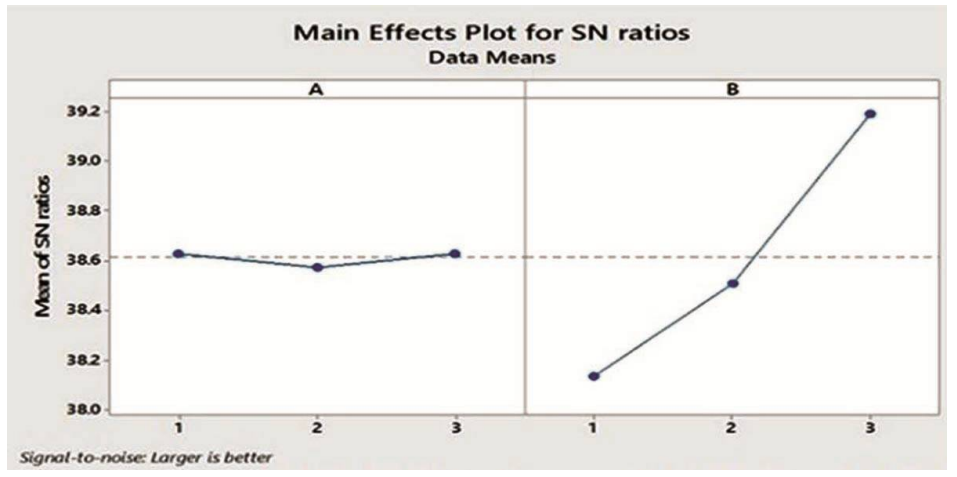

Figure 2. Main effects plot for SN ratios of A) type of oil phase, B) concentration of bentonite for entrapment efficiency

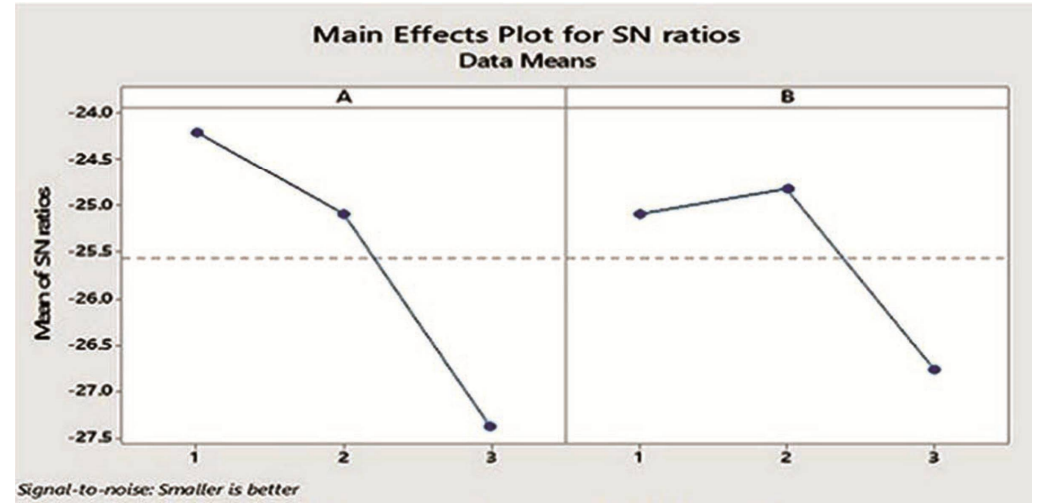

Figure 3. Main effects plot for $\mathrm{SN}$ ratios of A) type of oil phase, B) concentration of bentonite for percentage drug release.
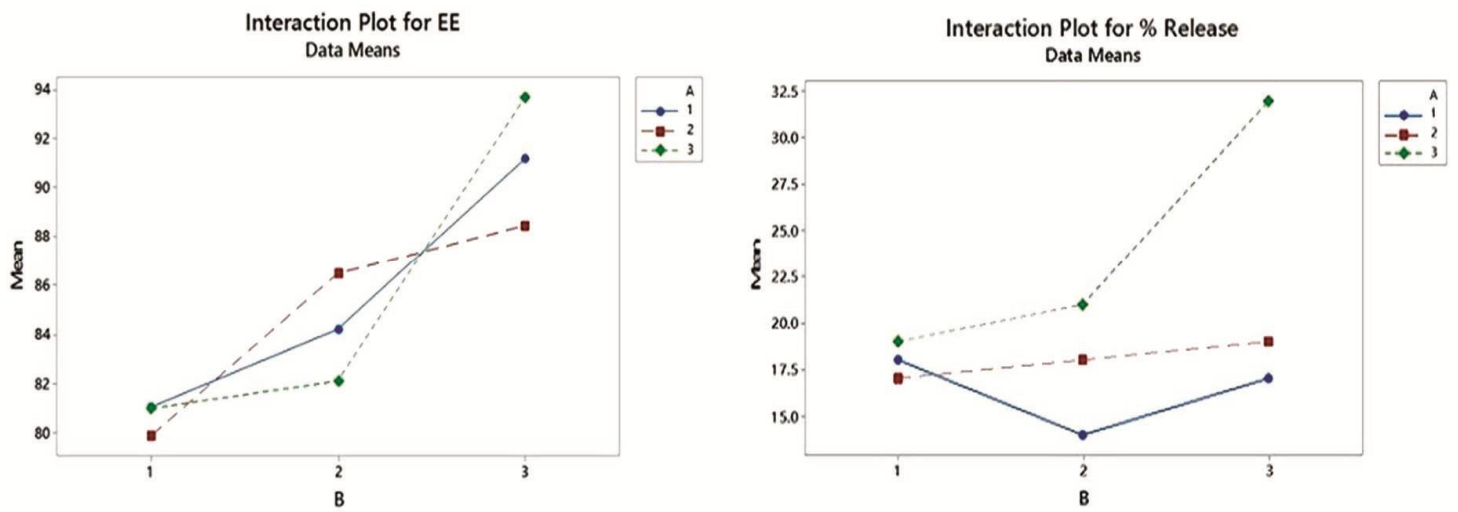

Figure 4. Interaction plots for Entrapment efficiency and percentage drug release. 
Surfactant based emulsion, SBE (classical emulsion) has shown maximum permeation Q8 of $925.8 \pm 0.02 \mu \mathrm{g} / \mathrm{cm}^{2}$, flux of $94.52 \pm 0.15 \mu \mathrm{g} / \mathrm{cm}^{2} / \mathrm{hr}$, permeability coefficient of $18.90 \pm 0.005 \mathrm{~cm} / \mathrm{hr} \times 10^{-3}$, lag time of $2 \pm 0.06 \mathrm{hr}$ and skin content of $3.71 \pm 0.52$ $\mathrm{mg} / \mathrm{g}$. Followed by PO3 with Q8 of $231.14 \pm 0.03$ $\mu \mathrm{g} / \mathrm{cm}^{2}$, flux of $18.83 \pm 0.05 \mu \mathrm{g} / \mathrm{cm}^{2} / \mathrm{hr}$, permeability coefficient of $3.76 \pm 0.004 \mathrm{~cm} / \mathrm{hr} \times 10^{-3}$, lag time of $1.8 \pm 0.025 \mathrm{hr}$ and skin content of $3.66 \pm 0.6 \mathrm{mg} / \mathrm{g}$. PS 3 which was optimized with Q8 of $166.70 \pm 0.15$ $\mu \mathrm{g} / \mathrm{cm}^{2}$, flux of $17.33 \pm 0.02 \mu \mathrm{g} / \mathrm{cm}^{2} / \mathrm{hr}$, permeability coefficient of $3.46 \pm 0.015 \mathrm{~cm} / \mathrm{hr} \times 10^{-3}$, lag time of $2.1 \pm 0.03 \mathrm{hr}$ and skin content of $4.01 \pm 0.24 \mathrm{mg} / \mathrm{g}$. The results of optimized formulation PO3 and PS3 in comparison with classical emulsion (SBE) are given in Table 3.

As the pickering emulsions are topical emulsions most of the drug should get deposited in to the epidermal layers of the skin than penetrating in to the systemic circulation. Thus, pickering emulsions are best suited for topical application and releasing the drug at target site.

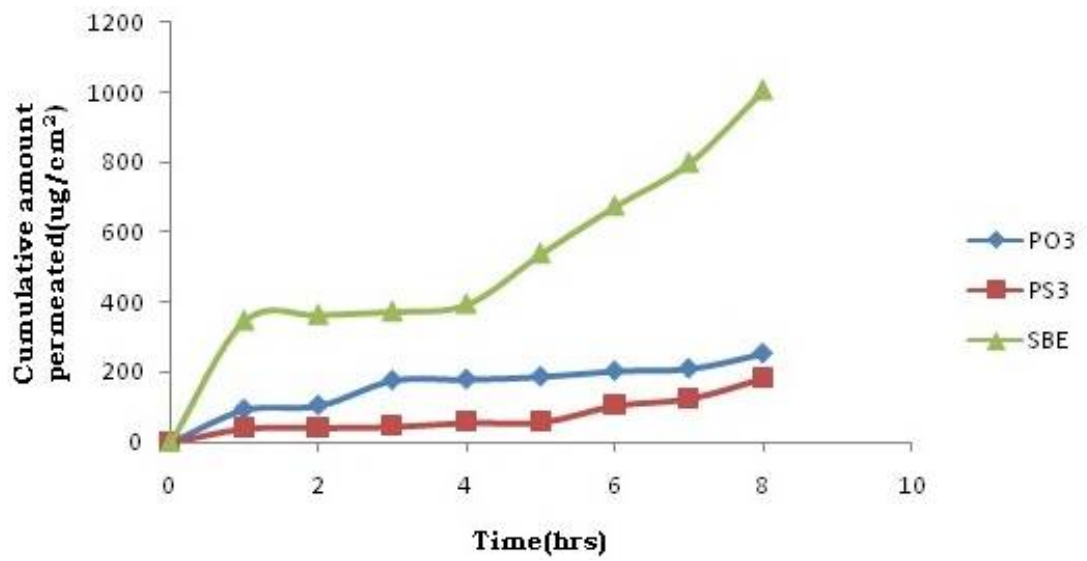

Figure 5. Cumulative amount permeated from Pickering emulsion formulations and surfactant-based emulsion (SBE).

Table 3. Characterization of optimized formulations of fluconazole Pickering emulsions.

\begin{tabular}{lccc}
\hline Parameter & PO3 & PS3 & SBE \\
\hline Particle size $(\mathrm{nm})$ & - & 758.0 & - \\
Zeta potential $(\mathrm{mV})$ & - & -48 & - \\
Entrapment efficiency $(\%)$ & $91.2 \pm 0.12$ & $93.7 \pm 0.42$ & $87.3 \pm 0.53$ \\
Drug content $(\%)$ & $99.61 \pm 0.2$ & $99.62 \pm 0.2$ & $98.25 \pm 0.3$ \\
Viscosity $(\mathrm{cps})$ & $569 \pm 0.3$ & $550 \pm 0.18$ & $600 \pm 0.25$ \\
flux $\left(\mu \mathrm{g} / \mathrm{cm}^{2} / \mathrm{hr}\right)$ & $18.83 \pm 0.05$ & $17.33 \pm 0.02$ & $94.52 \pm 0.15$ \\
Permeability co-efficient $(\mathrm{cm} / \mathrm{hr}) \times 10^{-3}$ & $3.76 \pm 0.004$ & $3.46 \pm 0.015$ & $18.90 \pm 0.005$ \\
Lag time $(\mathrm{hr})$ & $1.8 \pm 0.025$ & $2.1 \pm 0.03$ & $2 \pm 0.06$ \\
Skin deposition $(\mathrm{mg} / \mathrm{g})$ & $3.66 \pm 0.6$ & $4.01 \pm 0.24$ & $3.71 \pm 0.52$ \\
\hline
\end{tabular}

Note: Values represent Mean $\pm \mathrm{SD}, \mathrm{n}=3$

The results obtained from ex vivo permeation studies of the optimized batch were attempted to fit into model dependent kinetics. The regression coefficient $(r)$ values of zero order was 0.820 , first order (0.912) and Higuchi (0.717) of PS3 indicating that the drug release was found to follow first order release kinetics. Korsmeyer-Peppas equation, value of the exponent ' $n$ ' was found to be 0.684 indicating the drug release by Anomalous transport. 
The total amount of fluconazole absorbed in the skin is high for classical emulsion (SBE) than the Pickering emulsion. Pickering emulsions are "emulsifier-free", that is surfactants used as emulsifiers are not used instead fine solid particles are used, hence the penetration effect of surfactant is avoided. The presence of emulsifier/surfactant in classical emulsion (SBE) can modify the absorption process of the drug, as surfactants act as permeation enhancers. The droplet size has a definite effect on penetration of drug in to skin. The droplets of Pickering emulsions are surrounded by solid particles providing a rigid shell around the drops. ${ }^{11}$ This kind of structures can be viewed as an encapsulation system like microcapsules, allowing transport and providing sustained release. The rigidity of the shell around the oil droplets provides mechanical resistance; this gives stability better than the classical emulsion. It is thought that classical emulsion droplets break at surface and release the drug content in to the skin, whereas Pickering emulsion droplets may penetrate deeper in to skin. ${ }^{12}$ The oil droplets of Pickering emulsions resist droplet breaking but can deform since they are filled with liquid oil. Regarding highly lipophilic molecules, Pickering emulsions promote high storage in stratum corneum preventing skin penetration of molecules that should be retained at the skin surface or to slow the release of drug to the deeper skin layers. ${ }^{13}$

FTIR spectra peaks identified in the pure drug were $3120.61 \mathrm{~cm}^{-1}$, O-H stretching; $2960.53 \mathrm{~cm}^{-1}$, C-H stretching; $1618.17 \mathrm{~cm}^{-1}, \mathrm{C}=\mathrm{C} ; 1271.0 \mathrm{~cm}^{-1}, \mathrm{C}-\mathrm{F}$ stretching; $1413.72 \mathrm{~cm}^{-1}, \mathrm{C}-\mathrm{H}$ bending; $1519.80 \mathrm{~cm}^{-1}$, $\mathrm{C}=\mathrm{N}$ stretching; $1116.71 \mathrm{~cm}^{-1}, \mathrm{C}-\mathrm{O}$ stretching. The spectral peaks of formulation PS3 containing bentonite and sesame oil are $3400 \mathrm{~cm}^{-1}$, O-H stretching; $2962.11 \mathrm{~cm}^{-1}$, C-H stretching; 1633.76, $\mathrm{C}=\mathrm{C} ; 1454.38 \mathrm{~cm}^{-1}, \mathrm{C}=\mathrm{N}$ stretching; $1745.64 \mathrm{~cm}^{-1}$, $\mathrm{OH}$ group. It infers that there is no interaction between drug and the excipients. Therefore, the pure drug was not altered functionally.

Skin irritation study was performed and score attained was ' 0 ' indicating no erythema. The stability of optimised formulation was determined by performing stability for two months at room temperature. The optimised formulation (PS3) was found to be stable, but with slight variation in the entrapment efficiency and no creaming is observed. The emulsion was redispersable.

\section{CONCLUSIONS}

The production of $\mathrm{o} / \mathrm{w}$ emulsion without addition of surfactant was possible. Moreover, the incorporation of drug did not influence emulsion properties such as particle size and stability. Higher oil concentrations could not be used because of particle instability (coalescence). The formulation was optimized using Taguchi L9 OA experimental design. The formulation of sesame oil with $4 \%$ bentonite has shown better permeation than other formulations and the permeation is low than classical emulsion. Thus, Pickering emulsions are new drug penetration vehicles with specific behavior, they are well-suited either for targeting the stratum corneum or aimed at slow release of drug in to the deeper layers of the skin.

\section{REFERENCES}

1. Herbert, A.L., Martin, M.R. and Gilbert, S. 1996. In: Pharmaceutical Dosage Forms: Disperse systems (Lawrence H. Block), Marcel Dekker Inc, New York, Chapter 2, Volume 2, pp. 55-63.

2. Fabrizio, F. and Vincent, B. 2012. Pickering emulsion encapsulation for cosmetic industry. Bioencapsulation Innovations. 2, 20-21.

3. Dickinson E. 2009. In: Handbook of Hydrocolloids, CRC Press, New York, Chapter 2, pp. 23-47.

4. Charlier, C., Hart, E., Lefort, A., Ribaud, P., Dromer, F., Denning, D.W. and Lortholary, O. 2006. Fluconazole for the management of invasive candidiasis: where do we stand after 15 years? J. Antimicrob. Chemother. 57, 384-410.

5. Frelichowska, J., Bolzinger, M.A., Pelletier, J., Valour, J.P. and Chevalier, Y. 2009. Topical delivery of lipophilic drugs from o/w pickering emulsions. Int. J. Pharm. 371, 56-63.

6. Jani, R., Jani, K., Setty, C.M. and Patel, D. 2010. Preparation and evaluation of topical gel of valdecoxib. Int. J. Pharm. Sci. Drug Res. 2, 51-54.

7. Rakesh, P.P., Grishma, P., Patel, H. and Ashok, B. 2009. Formulation and evaluation of transdermal patch of aceclofenac. Int. J. Drug Deliv. 1, 41-51. 
8. Gangwar, S., Shivani, S.and Garg, G. 2010. Ethosomes: a novel tool for drug delivery through the skin. J. Pharm. Res. 3, 688-691.

9. Binks, B.P. and Kirkland, M. 2002. Interfacial structure of solid-stabilised emulsions studied by electron microscopy. Phys. Chem. Chem. Phys. 4, 3727-3733.

10. Barker, A.K., Naveed, A., Haji, M.S.K., Khalid, W., Tariq, M., Akhtar, R., Muhammad, I. and Haroon, K. 2011. Basics of pharmaceutical emulsions: a review. Afr. J. Pharm. Pharmacol. 5, 2715-2725
11. Bajaj, H., Bisht, S., Yadav, M., Singh, V. and Singh, M. 2011. Design and development of nevirapine loaded surfactant free chitosan microemulsion. Acta Pol. Pharm. 68, 981-988.

12. Frelichowska, J., Bolzinger, M.A., Valour, J.P., Mouaziz, H., Pelletier, J. and Chevalier, Y. 2009. Pickering w/o emulsions. Drug release and topical delivery. Int. J. Pharm. 368, 7-15.

13. Chin, L.S., Lim M. and Amal, R. 2011. Preparation and characterisation of perfluorodecalin in water pickering emulsion. Chemeca 2011: Engineering a Better World, Australia, pp. 2523-2532. 